\title{
Adenocarcinoma of Lacrimal Gland Non Otherwise Specified - A Case Report
}

\author{
Anita Syla Lokaj ${ }^{1}$, Kelmend Spahiu ${ }^{1 *}$ Blerta Rama $^{1}$ \\ ${ }^{1}$ Department of Ophthalmology, University Center Clinic of Kosova, Prishtina, Kosova
}

Received: March 25, 2018; Published: April 11, 2018

*Corresponding author: Kelmend Spahiu, Department of Ophthalmology, Prishtina, Kosova, Postal Code: 10000, Republic of Kosovo,

Email: kelmend.spahiu@hotmail.com, anitasylaj@live.com

\begin{abstract}
Background: Lacrimal gland adenocarcinoma is very rare tumor and highly agrressive.

Methods: A 86-year-old woman presented with a 5-month history of a painless mass in the right uppereyelid. The clinical presentation, workup, surgical approach, and pathological findings were reviewed.

Results: Incisional biopsy was performed toconfirm diagnosis. After histopathologicalconfirmation,exenteration was undergone and follows up further to oncology department.

Conclusion: Tumors of lacrimal gland are highly aggressive but fortunately are very rare. In invasive cases complete excision of tumor, or exenteration with adjuvant radiotherapy and chemotherapy is highly recommended.
\end{abstract}

Keywords: Lacrimal Gland; Adenocarcinoma NOS;Neoplasia; Exenteration

\section{Introduction}

Lacrimal gland tumors with epithelial origin are very rare and accounts about less than $5 \%$ of all biopsied orbital lesions [1]. The lacrimal gland is considered in some ways to be a type of minor salivary gland that shares histologic features with the major salivary glands. But many studies shows that lacrimal gland tumors and major salivary gland tumors are similar [2]. Until recently, primary adenocarcinoma of the lacrimal gland were not further subclassified, but now they can be divided into low-grade and high-grade malignancies. The most tumors of lacrimal gland are in epithelial origin, constituting approximately $50-60 \%$ of all benign tumors and $40-50 \%$ of all malignant tumors[3].

Approximately of $20 \%$ of all lacrimal gland bening tumor it is appropriate by pleomorphic adenoma. Malignant tumors of lacrimal gland are very rare, but very agressive and the most common form is adenoid cystic carcinoma. In the group of malignant tumors there is also included an unspecified group that numbers about 5 to 10 percent of very rare malignant tumors called adenocarcnimoa not otherwise specified (NOS)[3].In the absence of clear definition, special morphologic features, histopathological and imunohistochemical fenotipisation as in our paper not othervise specified adneocarinoam was determined. In this case we present a report and update on these different tumor types, withregard to their clinical presentation, histolopathology findings, immunohistochemical results and the best state treatment strategies for this rare tumor and more heterogeneous group of diseases[4].

\section{Case Report}

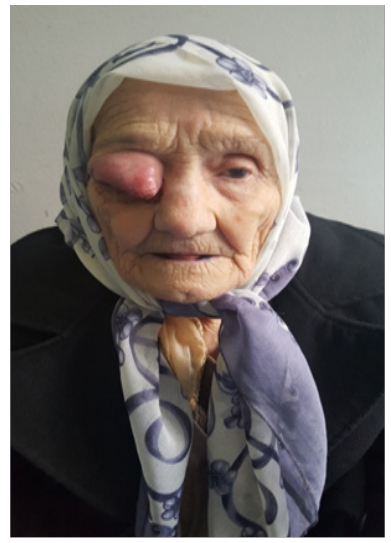

Figure 1: Stage when patient come in clinic.

An 86-year-old woman was referred to our clinic with a history of progressive, painless swelling over the right upper eyelid, for the 
past 5 months (Figure 1). She presented with ulceratet mass in this region of masses(Figure 3).Initially the tumor was too small, but it is grown rapidly(Figure 2). There were no systemic symptoms including loss of appetite, loss of weight, fever or a headache. No significant medical, surgical or family history could be elicited. An MRI scan of orbit and ocular area revealed a $45 \times 40 \times 35 \mathrm{~mm}$ solid mass in the upper eyelid with infiltration of rectus muscle but there was no intraocular, intraconal,extraconal , intracranial or paranasal sinus involvement. No bony erosions were noted.(Figure 4) MRI. In the appearance of the tumor we increase suspicion about Merkel cell carcinoma, so we decided to do incisional biopsy throw upper eyelid to confirm diagnosis.(Figure 5\& 6) Biopsy from the eyelid revealed a poorly differentiated carcinoma of epithelial origin with infiltrative neoplastic islands of epithelial malignant cell.

Figure 2: Proifle view of tumor.

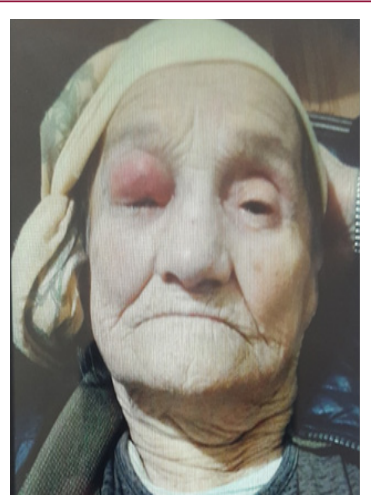

Figure 3: Inicial stage before 5-month.

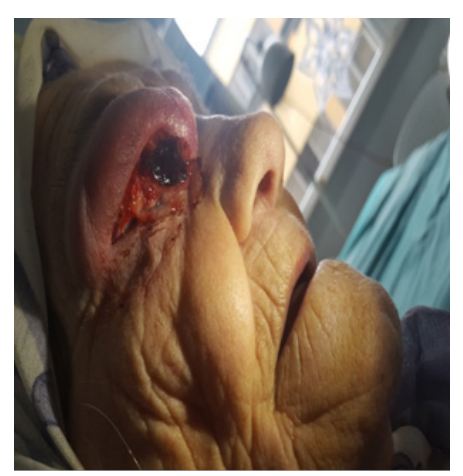

Figure 4: Ulcerated mass.

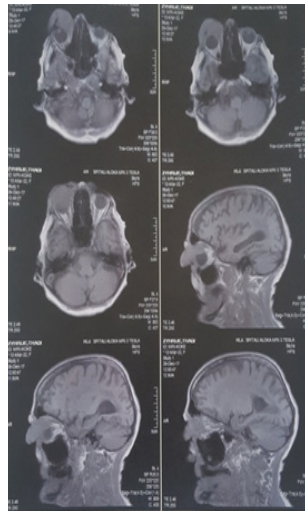

Figure 5: An MRI scan of the ocular space revealed a $45 \times 40 \times 35 \mathrm{~mm}$, malignant tumor on the right upper eyelid and lacrimal gland

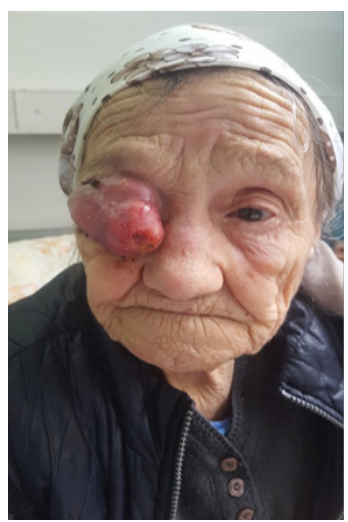

Figure 6: During operating day.

The neoplastic cells were large polygonal cell with vesicular nuclei, prominent nucleoli and amphophylic cytoplasm. But to make tumor phenotyping it was necessary to do immunohistochemistry examinations. On immunohistochemistry examination, cytokeratin 7 was positive, whereas cytokeratin 20 turned out to be negative. Based on histological analysis and immunohistochemical results we can conclud a poorly differentiated adenocarcinoma of lacrimal gland(grade 3).T Merkel cell carcinoma is excluded because absence of the phenotype CK20, CD56, Chromogranin A. We performed orbital exenteration under general anesthesia without any complications and instructed the patient to oncology department for further treatment with radiotherapy and chemotherapy.

\section{Disscussion}

Lacrimal gland fossa lesions may be divided into four categories: inflammatory, lymphoproliferative, epithelial lesions and metastatic tumors[5]. Out of epithelial lesions $55 \%$ are benign and $45 \%$ are malignant [6]. It is known that approximately $50 \%$ of lacrimal gland lesions are originated from epithelial elements and $50 \%$ are of nonepithelial origin [5] Epithelial tumors of the lacrimal gland are divided into low-grade and high-grade malignancies[7].Among the malignancies, most frequently present is adenoid cystic carcinoma (66\%), followed by a carcinoma-ex-pleomorphic adenoma (18\%), 
primary adenocarcinoma (9\%), and at least mucoepidermoid carcinoma (3\%)[6].Patient with glandula lacrimalis adenocarcinoma are associated with upper eyelid mass production which might be accompanied by symptoms such as exophthalmos, pseudoptosis, dystopia and in advanced disease reduced visual acuity [8].

However, the duration of the symptoms before first ophthalmic examination can vary; where for patients with pleomorphic adenoma is approximately 2 years, slow-growing tumors can persist up to 20 years, adenoid cystic carcinoma isapproximately 6 months and other malignant tumor can persist less than a year[9]. Comparing to the other malignant subtypes [10], painless or late clinical featured pain in the upper eyelid is more commonly present in adenocarnicoma patients, explaining neglected approach of the patient towardthisdisease and delayed decision for ophthalmology consultation, like ourpatient's case. This clinical feature and patient's history can lead into wide range of differential diagnosis of lacrimal gland lesions and eyelid malignant tumors. Therefore, basing on the eyelid mass protrusion, our initial approach was suspicion of the Merkel cell carcinoma. Meanwhile, we followed other possible lacrimal gland lesions, like lacrimal glandular epithelial tumors, lymphoid tumors, inflammatory diseases and miscellaneous diseseses; involving the lacrimal gland teratoma, granulocytic sarcoma, neurofibroma [11]To make the final diagnosis incisional biopsy was made; where immunohistochemical results revealed Lacrimal gland adenocarcinoma non-otherwise specified (NOS).

Adenocarcinoma NOS is used to distinguish nonspecitic carcinomas, because of the undistinguished histomorphology features characterize for the other, previously mentioned, more specific carcinoma[9].According to recent data adenocarcinoma NOS of lacrimal gland is very rare, therefore we have limited clinical trials, treatments, follow ups and prognosis[12].Only two cases with this diagnosis are represented; adenocarcinoma of the lacrimal gland presenting an abduction deficit [12] and adenocarcinoma NOS of the lower eyelid accessory lacrimal gland [4], diagnosis that also questioned the accessory lacrimal gland neoplasm classification [4].Our patient underwent orbital exenterating under general anesthetize and was sent to oncology department for further treatment with radiotherapy and chemotherapy.

\section{Conclussion}

It is known that the lacrimal glands can develop aggressive tumors like adenoid cystic carcinoma, carcinoma-ex-pleomorphic adenoma, mucoepidermoid carcinoma, primary adenocarcinoma or non-otherwise specified. Althought the clinical behavior, prognosis, and treatment of these tumors are still poorly defined, the glandula lacrimalis adenocarcinoma NOS is extremely rare. The diagnosis of the adenocarcinoma non otherwise specified NOS is made because of the absence of unifying morphologic, immunophenotypic, and molecular genetic findings. Basing on the absence of the clinical case and prognosis data, we assume that early recognition of this highly aggressive tumor may help to delineate its better management and good prognostic outcome. This case also represents the importance of the advanced immunohistochemical or other methods diagnostic approaches towards this group of disease, knowing that it also illustrates glandula lacrimal tumor classification challenges.

\section{References}

1. Touil A, El Abbassi S, Echchikhi Y, Maher M, Kebdani T, et al. (2017) Adenocarcinoma of the lacrimal gland: a case report. J Med Case Reports11(1):257.

2. Baek SO, Lee YJ, Moon SH, Kim YJ, Jun YJ (2012) Primary Adenocarcinoma of the Lacrimal Gland. Arch Plast Surg39(5):578-580.

3. An_Update_on_Tumors_of_the_Lacrimal_Gland/links/58eca6584585154f12544591/An-Update-on-Tumors-of-the-Lacrimal-Gland

4. Milman T, Iacob C, Lauer S, Feldman MD, Zhang PJL (2018) Adenocarcinoma, Not Otherwise Specified, Arising in Accessory Lacrimal Gland: A Diagnostic Challenge.Ocul Oncol Pathol4(2):67-72.

5. Eldesouky MA, Elbakary MA, Sabik S, Shareef MM (2014) Lacrimal fossa lesions: a review of 146 cases in Egypt. Clin Ophthalmol Auckl NZ8:1603-1609.

6. Baek SO, Lee YJ, Moon SH, Kim YJ, Jun YJ (2012) Primary adenocarcinoma of the lacrimal gland. Arch Plast Surg39(5):578-580.

7. Bernardini FP, Devoto MH, Croxatto JO (2008) Epithelial tumors of the lacrimal gland: an update. Curr Opin Ophthalmol19(5): 409-413.

8. Clauser L, Galie M, Tieghi R, Cavazzini L (2002) Adenocarcinoma of the lacrimal gland: report of a case. J Oral Maxillofac Surg60(3):318-321.

9. Von Holstein Sarah Linea, Coupland Sarah E, Briscoe Daniel, Le Tourneau Christophe, Heegaard Steffen (2012) Epithelial tumours of the lacrimal gland: a clinical, histopathological, surgical and oncological survey. Acta Ophthalmol91(3):195-206.

10. Von Holstein (2013) Tumours of the lacrimal gland. Epidemiological, Clinical and Genetic Characteristics. Acta Ophthalmological

11. Jung WS, Ahn KJ, Park MR, Kim JY, Choi JJ,et al. (2007) The Radiological Spectrum of Orbital Pathologies that Involve the Lacrimal Gland and the Lacrimal Fossa. Korean J Radiol8(4):336-342.

12. DeLuca CL, Zimmers RE (2017) Case Report: Adenocarcinoma of the Lacrimal Gland Presenting as an Abduction Deficit. Optom Vis Sci 94(12):1165-1169.
This work is licensed under Creative Commons Attribution 4.0 License

Submission Link: https://biomedres.us/submit-manuscript.php

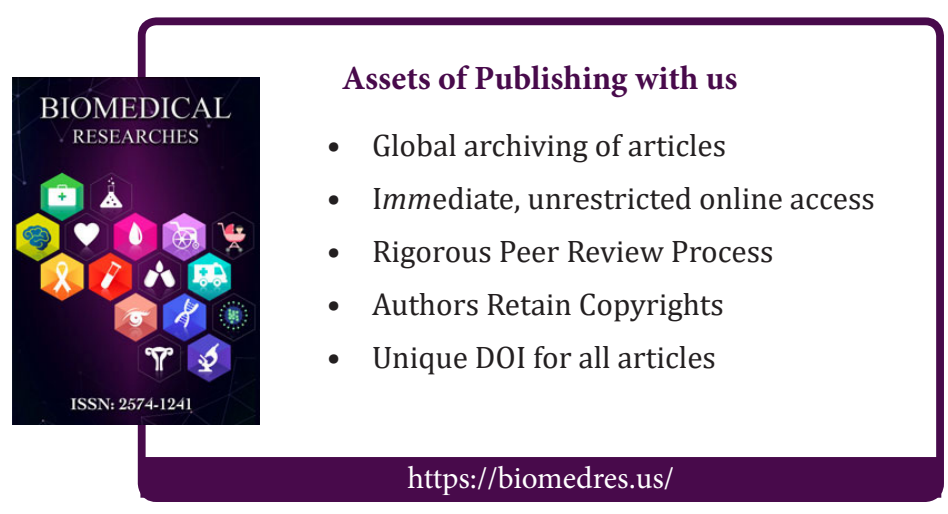

